\title{
Analysis Requested by Regulatory Agency
}

National Cancer Institute

\section{Source}

National Cancer Institute. Analysis Requested by Regulatory Agency. NCI Thesaurus.

Code C117751.

The analysis has been performed based on request from a regulatory agency. 